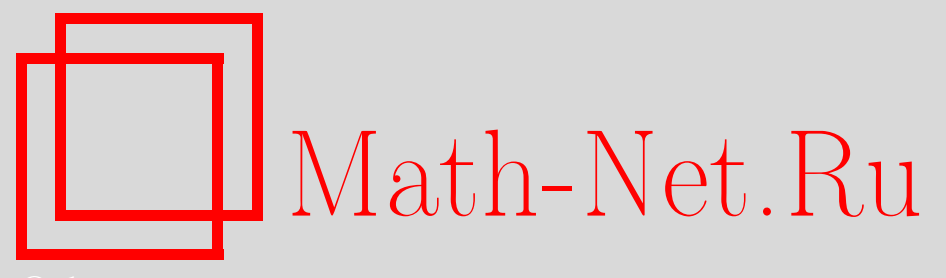

М. Бондаров, Попробуем решить иначе, Квант, 2020, номер 10, 26-31

DOI: https://doi.org/10.4213/kvant20201003

Использование Общероссийского математического портала Math-Net.Ru подразумевает, что вы прочитали и согласны с пользовательским соглашением http://www.mathnet.ru/rus/agreement

Параметры загрузки:

IP : 54.92 .164 .108

26 апреля 2023 г., 16:02:29

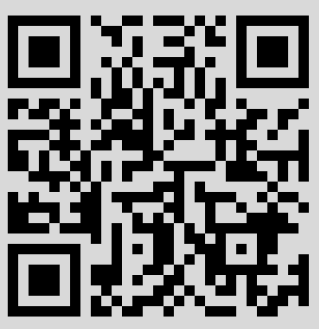




\section{Попробуем \\ решить иначе}

\section{М.БОНДАРОВ}

\section{Iresing} АК ИЗВЕСТНО, УНИВЕРСАЛЬНОГО

метода решения задач по физике не существует. Однако есть приемы, которые можно применить ко многим задачам. Среди них выделяются так называемые стандартные, знакомству с которыми обычно уделяется бо́льшая часть времени на уроках. Используя стандартные приемы, можно решить практически все задачи из различных задачников.

В то же время нередко бывает полезно заняться поиском альтернативных подходов к решению задачи. Случается, что такие поиски позволяют раскрыть новое содержание в условии, обнаружить некий скрытый смысл и тем самым глубже разобраться в физических явлениях, о которых говорится в задаче.

Рассмотрим некоторые из этих приемов на примере конкретных задач.

Задача 1. На гладком горизонтальном столе лежит призма массой $M$ с углом наклона $\alpha$, а на ней - призма массой $m$

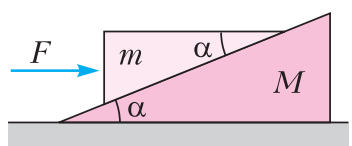

Pис. 1 (рис.1). На меньшую призму действует горизон тальная сила $F$, при этом обе призмь движутся вдоль стола как одно иелое (т.е. не изменяя взаимного расположения). Определите силу трения между призмами.

Стандартное решение. Стандартный подход к решению подобных задач хорошо известен. Вот его краткий путь: 1) делаем рисунок, на котором изображены тела, действующие на них силы, ускорения тел, оси координат; 2) записываем уравнения движения тел (второй закон Ньютона); 3) пишем вспомогательные уравнения (третий закон Ньютона, уравнения кинематики и т.п.).

DOI: https://doi.org/10.4213/kvant20201003
Итак, начнем с того, что изобразим на рисунках силы, действующие на каждую призму (лучше сделать два рисунка для каждого тела в отдельности). На верхнюю призму действуют сила тяжести $m \vec{g}$, сила $\vec{F}$ и две силы со стороны нижней призмы: сила реакции опоры (равная по величине силе

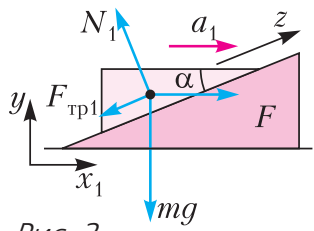

PИC. 2 нормального давления) $\vec{N}_{1}$ и сила трения покоя $\vec{F}_{\text {тр1 }}$ (рис.2). Направления трех первых сил определить не сложно, а вот выяснить, куда направлена сила трения, не так просто. Ясно, что она направлена вдоль поверхности соприкосновения призм, но куда именно вверх или вниз - без дополнительного исследования едва ли удастся определить. Придется выбрать одно из этих направлений произвольно (например, вниз, против оси z), а затем, дойдя до конечного ответа, нужно не забыть проанализировать полученный результат. После такого выбора однозначно определяются направления всех сил, действующих на нижнюю призму. Это сила тяжести $M \vec{g}$, сила реакции опоры со стороны стола $\vec{N}_{0}$, две силы со стороны верхней

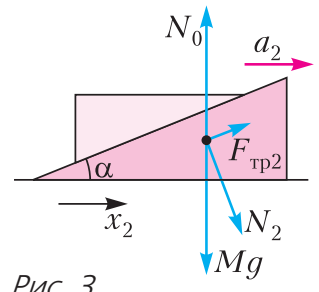

Pис. 3 призмы: сила нормального давления $\vec{N}_{2}$ и сила трения покоя $\vec{F}_{\text {тр2 }}$ - она направлена против $\vec{F}_{\text {тр1 }}$, т.е. вдоль оси $z$ (рис.3).

Рисунки 2 и 3 помогают нам записать уравнения второго закона Ньютона в векторной форме для каждой призмы:

$$
\begin{gathered}
\vec{F}+m \vec{g}+\vec{N}_{1}+\vec{F}_{\mathrm{Tp} 1}=m \vec{a}_{1}, \\
M \vec{g}+\vec{N}_{2}+\vec{F}_{\mathrm{Tp} 2}+\vec{N}_{0}=M \vec{a}_{2} .
\end{gathered}
$$

Эти векторные уравнения в проекциях на горизонтальную ось $(x)$ и вертикальную ось (y) с учетом третьего закона Ньютона: $\vec{N}_{1}=$ $=-\vec{N}_{2}, N_{1}=N_{2}=N ; \vec{F}_{\text {тр1 }}=-\vec{F}_{\text {тр } 2}, F_{\text {тр1 }}=F_{\text {тр2 }}=$ $=F_{\text {тр }}$ и того факта, что тела движутся как единое целое: $a_{1}=a_{2}=a$, принимают вид

$$
\begin{gathered}
F-F_{\text {тр }} \cos \alpha-N \sin \alpha=m a, \\
F_{\text {тр }} \cos \alpha+N \sin \alpha=M a, \\
N \cos \alpha-m g-F_{\text {тр }} \sin \alpha=0 .
\end{gathered}
$$


Таким образом, получена система уравнений, из которой можно определить искомую силу трения:

$$
F_{\text {тр }}=\frac{M}{M+m} F \cos \alpha-m g \sin \alpha .
$$

Ну что ж, конечный результат получен. Является ли этот ответ полным и можно ли из него понять, угадано ли нами направление силы трения? Вспомним наше предположение: сила трения, действующая на верхнюю призму, направлена против оси $z$. Из анализа последнего выражения следует, что выбранное нами направление силы трения совпадает с истинным, если

$$
\begin{aligned}
\frac{M}{M+m} F \cos \alpha-m g \sin \alpha & >0 \text {, т.е. } \\
F & >\frac{M+m}{M} m g \operatorname{tg} \alpha .
\end{aligned}
$$

В противном случае, если

$$
F<\frac{M+m}{M} m g \operatorname{tg} \alpha,
$$

направления сил трения $\vec{F}_{\text {тр1 }}$ и $\vec{F}_{\text {тр2 }}$ меняются на противоположные, но их модули можно рассчитывать по единой формуле:

$$
F_{\text {тр }}=\left|\frac{M}{M+m} F \cos \alpha-m g \sin \alpha\right| .
$$

Это и есть ответ к задаче.

Подводим итог этому способу решения. Можно ли было осуществить его короче? На первый взгляд, вроде бы, нет. Но на самом деле такой путь имеется, и состоит он в том, чтобы объединить тела, заменив их одним с общей массой и общим ускорением. Это позволит не учитывать силы взаимодействия между призмами, которые станут внутренними и автоматически исчезнут из уравнений. Правда, тем самым пропадет искомая величина. Что ж, дополнительно придется записать еще одно уравнение движения для одной из призм. А чтобы избежать попадания в него неизвестной силы, воспользуемся еще одним приемом: удачным выбором оси координат.

\section{Альтернативное ре-} шение. Рассмотрим обе призмы как одно тело, масса которого равна $M+m$, движущееся горизонтально с ускорением $\vec{a}$ (рис.4). На него действуют три

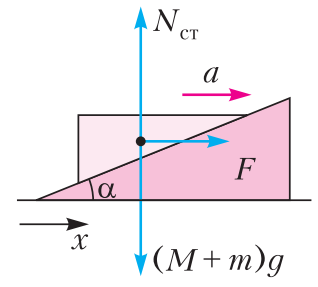

Pис. 4 силы: сила тяжести $(M+m) \vec{g}$, сила нормальной реакции со стороны стола $\vec{N}_{\text {ст }}$ и горизонтальная сила $\vec{F}$. Второй закон Ньютона для этого объединенного тела в проекции на горизонтальную ось $x$ принимает вид

$$
F=(M+m) a .
$$

Искомую силу трения проще всего найти, рассмотрев верхнюю призму (см. рис.2). Запишем для этой призмы второй закон Ньютона в проекциях на ось $z$ :

$$
F \cos \alpha-F_{\text {тр }}-m g \sin \alpha=m a \cos \alpha .
$$

Как видим, выбор оси, действительно, оказался удачным. Поскольку вектор силы $\vec{N}_{1}$ перпендикулярен оси $z$, его проекция на эту ось равна нулю. Таким образом, имеем систему из двух уравнений с двумя неизвестными, откуда легко находим искомую силу трения:

$$
F_{\text {тр }}=\frac{M}{M+m} F \cos \alpha-m g \sin \alpha .
$$

Подводим итог. Примененные нами приемы - объединение тел в единое целое и удачный выбор оси, на которую проецируется уравнение второго закона Ньютона, позволили значительно упростить решение данной задачи.

Задача 2. На гладкой горизонтальной поверхности находится горка, масса которой $M=8 \kappa 2$, а высота $h=1$ м (рис.5). Нa вершину горки положили тело массой $m=2$ кг, которое, скатившись, упруго ударяется о пружину, закрепленную неподвижно, и вновь поднимается нагорку. Найдите максимальную высоту

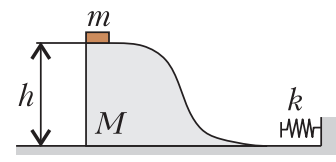

Рис. 5

H, на которую поднимется тело в этом случае. Трение в системе отсутствует.

Стандартное решение. Заметим, что в данном случае движения тела по горке и самой горки по горизонтальной поверхности не являются равноускоренными. Поэтому динамический подход в данной задаче применить не удастся. Ничего страшного, в нашем распоряжении имеются законы сохранения. Их можно использовать, поскольку трение в системе отсутствует и в горизонтальном направлении никаких внешних воздействий нет, не считая кратковременного соприкосновения тела с пружиной. 
Разобьем решение на три этапа: 1) спуск тела с горки; 2) взаимодействие тела с пружиной; 3) въезд тела на горку.

1) Пусть скорость тела после спуска с горки равна $v_{1}$, а скорость самой горки в этот момент равна $v_{2}$. Запишем законы сохранения импульса и механической энергии для системы «горка - тело»:

$$
\begin{gathered}
0=m v_{1}-M v_{2}, \\
m g h=\frac{m v_{1}^{2}}{2}+\frac{M v_{2}^{2}}{2} .
\end{gathered}
$$

Из этих уравнений определим скорости горки и тела при его движении до пружины:

$$
v_{2}=\sqrt{\frac{2 m^{2} g h}{M(M+m)}}, v_{1}=\sqrt{\frac{2 M g h}{M+m}} .
$$

2) Взаимодействие тела с пружиной приведет к тому, что вектор его скорости изменит направление на противоположное, оставив прежней свою величину $v_{1}$.

3) Наконец, отскочив от пружины, тело массой $m$ догонит горку, заедет на нее и в точке наивысшего подъема будет вместе с ней двигаться относительно земли со скоростью $v_{3}$. Законы сохранения теперь примут такой вид:

$$
\begin{gathered}
m v_{1}+M v_{2}=(M+m) v_{3}, \\
\frac{m v_{1}^{2}}{2}+\frac{M v_{2}^{2}}{2}=\frac{(M+m) v_{3}^{2}}{2}+m g H .
\end{gathered}
$$

Из этой системы уравнений получаем

$$
H=h\left(\frac{M-m}{M+m}\right)^{2}=0,36 \text { м. }
$$

Альтернативное решение. Энергия системы «горка - тело» будет оставаться неизменной в любой момент времени, когда пружина не деформирована. В начальный момент энергия $E_{1}$ системы равна потенциальной энергии тела (за ноль потенциальной энергии принята энергия на уровне плоскости):

$$
E_{1}=m g h .
$$

Из закона сохранения импульса следует, что после того как тело покинет горку, но еще не коснется пружины, тело и горка будут иметь равные по модулю импульсы $p$. В это время энергия системы $E_{2}$ состоит из суммы кинетических энергий горки и тела:

$$
E_{2}=\frac{p^{2}}{2 M}+\frac{p^{2}}{2 m} .
$$

Когда тело, отскочив от пружины, догонит горку и поднимется на максимальную высоту, система «горка - тело» будет иметь импульс $2 p$, а ее энергия станет равной

$$
E_{3}=\frac{(2 p)^{2}}{2(M+m)}+m g H .
$$

Поскольку выполняется закон сохранения механической энергии, то $E_{1}=E_{2}=E_{3}$, или

$$
m g h=\frac{p^{2}}{2 M}+\frac{p^{2}}{2 m}=\frac{(2 p)^{2}}{2(M+m)}+m g H .
$$

Отсюда находим искомую высоту:

$$
H=h\left(\frac{M-m}{M+m}\right)^{2} .
$$

Как видим, неявное использование закона сохранения импульса и не часто встречающаяся запись формуль кинетической энергии $E_{\text {к }}=\frac{p^{2}}{2 m}$ позволили упростить решение задачи.

Замечание. В некоторых задачах полезно учесть соотношение между заданными величинами, что иногда позволяет существенно сократить процесс решения. Так, в нашем случае можно было прийти к верному ответу практически устно, заметив, что $M=4 \mathrm{~m}$. Действительно, из последней формулы для кинетической энергии следует, что после соскальзывания кинетическая энергия тела $E_{\text {кт2 }}$ будет составлять 4/5 от общей энергии системы $E_{1}$, т.е. $E_{\text {кт2 }}=\frac{4}{5} m g h$. Выразим далее через $E_{1}$ общую кинетическую энергию $E_{\text {к3 }}$ системы «горка - тело» в момент достижения телом высоты $H$. Поскольку импульс системы вдвое больше импульса тела после отскока от пружины, a eе масса больше массы тела в 5 раз, то

$$
E_{\text {к } 3}=\frac{2^{2}}{5} E_{\text {кт } 2}=\frac{2^{2}}{5} \cdot \frac{4}{5} m g h=\frac{16}{25} m g h .
$$

Итак, в начале движения полная энергия системы равнялась $m g h$, в конце кинетическая энергия системы составляла $\frac{16}{25} \mathrm{mgh}$. Значит, на долю потенциальной энергии в этот момент приходилось $\frac{9}{25} m g h$. Таким образом, искомая высота равна

$$
H=\frac{9}{25} h=0,36 \text { м. }
$$


Задача 3. С поверхности земли подброшен вертикально вверх небольшой шарик с начальной скоростью $v_{0}=5 \mathrm{~m} / \mathrm{c} . B \mathrm{mom}$ момент когда он достиг верхней точки, снизу с того же места подброшен точно такой же шарик с такой же начальной скоростью. При столкновении шарики слипаются и движутся далее как одно челое. Определите промежуток времени $t$, в течение которого первый шарик находился в полете от момента броска до момента соприкосновения с поверхностью земли. Сопротивлением воздуха можно пренебречь. Ускорение свободного падения примите равным $g=10 \mathrm{~m} / \mathrm{c}^{2}$.

Стандартное решение. Выберем систему отсчета с началом на поверхности земли и координатной осью $y$, направленной вертикально вверх. Уравнения движения шариков имеют вид

$$
\begin{gathered}
y_{1}(t)=v_{0} t-\frac{g t^{2}}{2}, \\
y_{2}(t)=v_{0}\left(t-t_{0}\right)-\frac{g\left(t-t_{0}\right)^{2}}{2},
\end{gathered}
$$

где $t_{0}=\frac{v_{0}}{g}-$ время подъема первого шарика до верхней точки. Из равенства $y_{1}\left(t_{1}\right)=y_{2}\left(t_{2}\right)$ находим, что промежуток времени $t_{1}$ от момента подбрасывания первого шарика до столкновения шариков равен

$$
t_{1}=\frac{3 v_{0}}{2 g},
$$

а высота $h$, на которой произойдет столкновение, составляет

$$
h=\frac{3 v_{0}^{2}}{8 g} .
$$

Непосредственно перед столкновением скорости каждого из шариков по величине равны $v=v_{0} / 2$, но направлены в противоположные стороны. По закону сохранения импульса сразу после столкновения скорость слипшихся шариков равна нулю. Время их свободного падения на землю с высоты $h$ равно

$$
t_{2}=\sqrt{\frac{2 h}{g}}=\frac{v_{0}}{2 g} \sqrt{3} .
$$

Общее время полета первого шарика (т.е. искомый промежуток времени) равно

$$
t=t_{1}+t_{2}=\frac{v_{0}}{2 g}(3+\sqrt{3}) \approx 1,2 \mathrm{c} .
$$

Альтернативное решение. Как и в первом способе решения, определим сначала время полета первого шарика до верхней точки: $t_{0}=v_{0} / g$. Рассмотрим далее систему из двух шариков как единое целое. Центр масс этой системы находится на высоте $H / 2$ и имеет скорость $v_{0} / 2$. Здесь $H-$ максимальная высота подъема первого шарика, которую легко найти из закона сохранения энергии:

$$
\frac{m v_{0}^{2}}{2}=m g H \text {, и } H=\frac{v_{0}^{2}}{2 g} .
$$

У равнение движения центра масс от момента слипания шариков до момента их падения в течение времени $T$ имеет вид

$$
-\frac{H}{2}=\frac{v_{0}}{2} T-\frac{g T^{2}}{2},
$$

или

$$
T^{2}-\frac{v_{0}}{g} T-\frac{v_{0}^{2}}{2 g^{2}}=0 .
$$

Положительный корень этого уравнения равен

$$
T=\frac{v_{0}}{2 g}(1+\sqrt{3})
$$

Таким образом, общее время полета равно

$$
t=t_{0}+T=\frac{v_{0}}{2 g}(3+\sqrt{3}) .
$$

Обратите внимание, что, решая первым способом, мы попутно узнали некоторые подробности движения (например, время движения шариков до столкновения и высоту, на которой они столкнулись). Зато второй способ - метод центра масс - оказался значительно короче.

Задача 4. Груз висит на пружине жесткостью $k=60 \mathrm{H} /$ м. Какую надо совершить работу, итобы растянуть пружину еще на $x_{1}=2 \quad c \mu ?$

Стандартное решение. При растяжении пружины изменяются две потенциальные энергии: самой пружины - она увеличивается на $\Delta E_{\text {п1 }}=\frac{k\left(x_{0}+x_{1}\right)^{2}}{2}-\frac{k x_{0}^{2}}{2}$ и груза в поле тяготения - его энергия уменьшается на $\Delta E_{\text {п2 }}=m g x_{1}$, где $x_{0}-$ начальная деформация пружины, $m$ - масса груза. Искомая работа пойдет на изменение этих потенциальных энергий:

$$
A=\frac{k\left(x_{0}+x_{1}\right)^{2}}{2}-\left(m g x_{1}+\frac{k x_{0}^{2}}{2}\right)
$$


При этом в начальном положении груз находился в равновесии:

$$
m g=k x_{0} .
$$

Из этих выражений получим

$$
A=\frac{k x_{1}^{2}}{2}=12 \text { мДж. }
$$

Задача решена, но трудно не заметить, что конечное выражение для работы имеет тот же вид, как если бы происходило растяжение недеформированной пружины на величину $x_{1}$. Попробуем разобраться, в чем причина такого совпадения.

Альтернативное решение. Изобразим график зависимости силы упругости $F$, возникающей в пружине, от ее удлинения $x$ (рис.6).

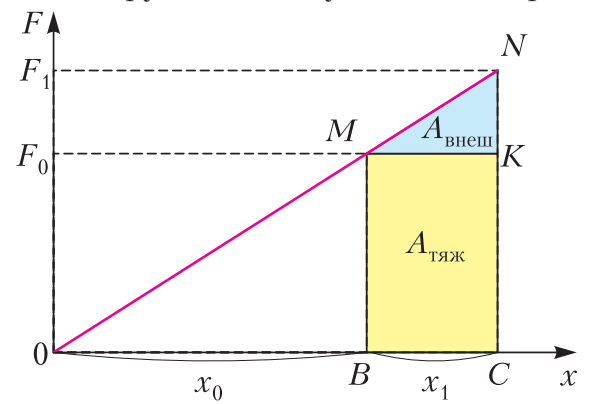

PทC. 6

Первоначально груз растянул пружину на величину $x_{0}$, поэтому

$$
m g=F_{0}=k x_{0} .
$$

При растяжении пружины еще на величину $x_{1}$ она совершает отрицательную работу, модуль которой численно равен площади трапеции $B M N C$. В то же время из графика видно, что работа силы тяжести $A_{\text {тяж }}$ (выделено желтым) и работа искомой внешней силы $A_{\text {внеш }}$ (выделено синим) в сумме дают то же значение. По условию требуется определить только $A_{\text {внеш }}$ Ее легко найти из графика:

$$
A_{\text {внеш }}=\frac{\left(F_{1}-F_{0}\right) x_{1}}{2} .
$$

Но $F_{1}-F_{0}=k x_{1}$, поэтому

$$
A_{\text {внеш }}=\frac{k x_{1}^{2}}{2} .
$$

Замечание. Давайте снова посмотрим на график. Если перенести выделенный синим треугольник в начало координат, то нетрудно видеть, что его площадь будет численно равна работе силы упругости при ее растяжении из недеформированного состояния на величину $x_{1}$. Другими словами, полезно иметь в виду, что при смещении подвешенного груза от положения равновесия действие двух сил - тяжести и упругости - можно заменить на действие одной силь упругос$m u$, деформация которой отсчитывается от положения равновесия.

Задача 5. На наклонной плоскости с углом а находится кубик (рис. 7). К кубику

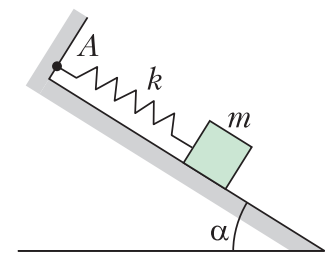

PUC. 7 прикреплена легкая пружина, другой конеи которой закреплен в неподвижной точке А. В исходном состоянии кубик удерживается в положении, при котором пружина не деформирована. Кубик отпускают без начальной скорости. Определите максимальную скорость кубика в прочессе движения. Масса кубика m, жесткость пружинь $k$, коэффициент трения $\mu(\mu<\operatorname{tg} \alpha)$.

Стандартное решение. В процессе движения на кубик действуют (рис.8): сила тяже-

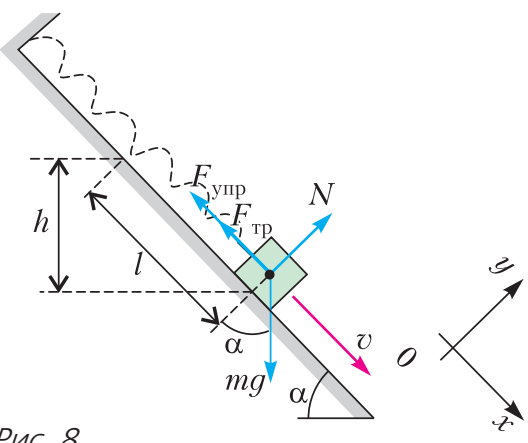

сти $m \vec{g}$, направленная вертикально вниз; сила нормальной реакции опоры $\vec{N}$, направленная перпендикулярно наклонной плоскости; сила трения скольжения $\vec{F}_{\text {тр }}$, направленная вдоль наклонной плоскости вверх; сила упругости пружины $\vec{F}_{\text {упр }}$, направленная также вдоль наклонной плоскости вверх (предполагается, что ось пружины параллельна наклонной плоскости).

По условию в начальный момент пружина не деформирована. Когда кубик отпускают, он начинает двигаться прямолинейно по наклонной плоскости вниз. При этом скорость кубика увеличивается и в некоторый момент времени достигает искомого максимального значения $v$. Пусть к этому моменту кубик 
прошел вдоль наклонной плоскости путь $l$. Значит, деформация пружины при этом также равна $l$. Кроме того, смещение кубика по вертикали вниз будет равно $h=l \sin \alpha$. Если считать потенциальную энергию кубика в поле тяжести в этом положении равной нулю, то приращение механической энергии кубика за время, прошедшее с момента начала движения, будет равно

$$
\Delta E=\frac{m v^{2}}{2}+\frac{k l^{2}}{2}-m g l \sin \alpha .
$$

С другой стороны, это приращение должно быть равно суммарной работе неконсервативных сил, действующих на кубик. Сила нормальной реакции опоры работы не совершает, а работа силы трения скольжения равна $A_{\text {тр }}=-F_{\text {тр }} l$, причем $F_{\text {тр }}=\mu N$. Тогда

$$
\frac{m v^{2}}{2}+\frac{k l^{2}}{2}-m g l \sin \alpha=-\mu N l \text {. }
$$

Запишем для кубика уравнения второго закона Ньютона в проекциях на оси $x$ и $y$ для момента времени, когда скорость кубика максимальна (ускорение кубика при этом равно нулю):

$$
\begin{gathered}
m g \sin \alpha-F_{\text {упр }}-F_{\text {тр }}=0, \\
N-m g \cos \alpha=0,
\end{gathered}
$$

где $F_{\text {упр }}=k l, F_{\text {тр }}=\mu N$. Отсюда

$$
l=\frac{m}{k} g(\sin \alpha-\mu \cos \alpha) \text {. }
$$

Окончательно получим

$$
v=\sqrt{\frac{m}{k}} g(\sin \alpha-\mu \cos \alpha) .
$$

Альтернативное решение. После решения задачи в общем виде полезно задуматься над ответом. Не правда ли, в нем проявляются знакомые черты? Ну, конечно, искомая скорость равна произведению двух хорошо известных величин. Один из сомножителей это ускорение $a$ тела, скатывающегося с наклонной плоскости:

$$
a=g(\sin \alpha-\mu \cos \alpha) .
$$

Другой сомножитель - величина, обратная циклической частоте $\omega$ гармонических колебаний груза на пружине:

$$
\frac{1}{\omega}=\sqrt{\frac{m}{k}} .
$$

Случайно ли такое совпадение? Скорее всего, нет, ведь обе величины имеют непосред- ственное отношение к процессам, происходящим в нашей задаче. Правда, формула для ускорения подходит лишь в случае отсутствия действия на тело силы упругости пружины. Тогда как формулу циклической частоты привычно применяют для колебаний груза на пружине без трения. У нас же сила упругости пружины отсутствует только в начальный момент движения, когда ускорение тела максимально. Стоп! Вот мы и наткнулись на подсказку. Существует же формула связи максимальной скорости $v_{m}$ и максимального ускорения $a_{m}$ при гармонических колебаниях:

$$
a_{m}=\omega v_{m} .
$$

Выходит, если подставить в нее выражения для $a$ и $\omega$, получится верное значение для максимальной скорости.

А возможно ли применение формулы, связывающей $a_{m}$ и $v_{m}$, в данной задаче? Вспомним происхождение этой формулы. Как известно, она выводилась из уравнения зависимости координаты колеблющегося тела от времени: $x=x_{m} \cos \left(\omega t+\varphi_{0}\right)$. А само это уравнение оказалось решением дифференциального уравнения (второго закона Ньютона) для колебательного движения: $x^{\prime \prime}+\omega t=0$, где $\omega=\sqrt{\frac{k}{m}}-$ частота для колебаний груза на пружине.

Итак, направление поиска известно: надо записать аналогичное уравнение в нашем случае и сравнить с уравнением колебаний. Из анализа стандартного решения следует:

$$
m g \sin \alpha-F_{\text {упр }}-F_{\text {тр }}=m a \text {. }
$$

Но $a=x^{\prime \prime}, F_{\text {упр }}=k x(x-$ удлинение пружины в произвольный момент времени), $F_{\text {тр }}=\mu m g \cos \alpha$. Тогда получим

$$
m g \sin \alpha-k x-\mu m g \cos \alpha=m x^{\prime \prime},
$$

или

$$
x^{\prime \prime}+\frac{k}{m} x+g(\mu \cos \alpha-\sin \alpha)=0 .
$$

Это уравнение отличается от уравнения колебаний лишь на константу $C=$ $=g(\mu \cos \alpha-\sin \alpha)$. А значит, в нашем случае циклическая частота и соотношение между максимальными величинами ускорения и скорости имеют тот же вид, что и для гармонических колебаний. 